adequate counseling. The adoption of a contraceptive method and the number of pregnancies were not different between the two groups. The history of use of embryotoxic/teratogenic drugs was associated with a higher frequency of adequate counseling $(79.6 \%$ vs $41.5 \%, p=0.001)$. According to the patients, adequate counseling was given by the rheumatologist in $75.5 \%$ of the cases $(p=0.001)$. Conclusions: Adequate preconceptional counseling in our patients with ARDs at the reproductive stage is deficient. A multidisciplinary strategy is required to improve the frequency and quality of preconception counseling in patients with ARDs.

Disclosure of Interest: None declared

DOI: 10.1136/annrheumdis-2017-eular.6475

\section{FRI0280 ASSOCIATION BETWEEN INSULIN RESISTANCE, SUBCLINICAL ATHEROSCLEROSIS AND ACTIVITY/DAMAGE STATUS IN SLE PATIENTS}

H. Sánchez-Pérez ${ }^{1}$, B. Tejera Segura ${ }^{1}$, M.A. Gonzalez-Gay ${ }^{2}$, I. Ferraz-Amaro ${ }^{1}$ ${ }^{1}$ Rheumatology, Hospital Universitario de Canarias, la Laguna; ${ }^{2}$ Rheumatology, Hospital Marques de Valdecilla, Santander, Spain

Background: Insulin resistance (IR) may contribute to an increase in cardiovascular risk in general population as well as in Systemic Lupus Erythematosus (SLE) patients.

Objectives: The aim of this study was to examine the association between IR and disease activity, disease characteristics, drug exposure and subclinical atherosclerosis in patients with SLE.

Methods: Cross-sectional study that encompassed 87 SLE patients and 82 sex-matched controls. IR by homeostatic model assessment (HOMA2), insulin, $\mathrm{C}$-peptide serum levels and lipid profile were assessed in both groups. Activity (SLEDAI), severity (Katz) and damage (SLICC) scores, carotid intima-media thickness (cIMT) and carotid plaques (ultrasound) were assessed in SLE patients. A multivariable regression analysis, adjusted for IR related factors, was performed to evaluate the differences between groups in IR indexes and, in SLE patients, the interrelation between IR and disease activity/characteristics as well as subclinical atherosclerosis.

Results: Median disease duration was 16 years (IQR 9-21). Body mass index, abdominal circumference, hypertension or dyslipidemia did not differ between groups. According to the SLEDAI score, $40 \%$ of patients were in no activity, while $32,21,18$ and $1 \%$ were in mild, moderate, high and very high activity respectively. HOMA-IR-C-peptide (beta coefficiente 0.53 , [95\% Cl 0.25-0.82], $p=0.00$ ) was increased in SLE patients when compared to controls, as well as HOMA \%B C peptide levels (beta coef. $35,95 \% \mathrm{Cl} 18-52, p=0.00$ ). Similarly, insulin sensitivity estimated through HOMA-S\% was inferior in SLE patients (-beta coef. - $37,95 \%$ $\mathrm{Cl}-63-11, \mathrm{p}=0.01)$. This diferences remained significant even after adjustment for IR related factors.

SLICC damage index was clearly asociated with IR indexes; higher index values were related to higher HOMA-IR-C-peptide (beta coef. 37, [95\% Cl 16-57], $p=0.00$ ) and lower HOMA-S\% levels (beta coef. 30\%, [95\% Cl -47-14], p=0.00). Katz severity index showed correlation with HOMA-IR-C-peptide (beta coef.-5, $[95 \% \mathrm{Cl}-11-0], \mathrm{p}=0.04)$. These associations remained significant after adjustment for age, gender, smoking, hypertension and dyslipidemia, and, in relation with the SLICC index, also after adjustment for prednisone intake. SLEDAI activity index was not related to IR indexes.

The use of prednisone was positively associated with HOMA-IR both when considered binary (beta coef $47,[95 \% \mathrm{Cl} 31-63], \mathrm{p}=0.00$ ) and continous (beta coef 2 [95\% Cl 0-5] per mg, $p=0.03$ ). Hydroxycloroquine (or other drugs) use was not associated with IR indexes, neither were disease duration, antiDNA titers and complement serum levels.

Carotid plaques were found in $20 \%$ of the SLE patients. The presence of carotid plaques was correlated with a higher HOMA-IR-C-peptide (OR 3.15 [95\% IC 1.17-8.51], $\mathrm{p}=0.02)$, and a higher cIMT value was associated with a lower HOMAIR-S\%>C-peptide (beta coef. 0.98 [95\% Cl 0.96-0.99], $p=0.03$ ). Nevertheless, after adjustment for cardiovascular risk factors this relation was lost.

Conclusions: Activity and damage indexes in SLE patients are independently related to the development of IR. IR is associated with subclinical carotid atherosclerosis in SLE patients on the univariate analysis.

Disclosure of Interest: None declared

DOI: 10.1136/annrheumdis-2017-eular.2367

\section{FRI0281 UTILITY OF MORNING SAMPLE OF URINE PROTEIN/CREATININE RATIO FOR ASSESSMENT OF PROTEINURIA IN PATIENTS WITH LUPUS NEPHRITIS}

H. Zhang, J. Liang, B. Hua, L. Sun. Department of Rheumatology and Immunology, The Affiliated Drum Tower Hospital of Nanjing University Medical School, Nanjing, China

Background: Proteinuria is the principal urinary biomarker for the screening of Lupus nephritis (LN) and for monitoring disease progression. 24-hour urine collection has been the foundation for monitoring disease activity in patients with $\mathrm{LN}$, but collections are often inaccurate and inconvenient. The European League Against Rheumatism and American College of Rheumatology have recommended the urine protein/creatinine ratio (UPCR) for use in management of LN.
Objectives: We aimed to evaluate the diagnostic accuracy of the morning sample of UPCR compared with 24-hour urine collection for the detection of proteinuria and to determine the UPCR for different proteinuria ranges in patients with LN.

Methods: Three hundred and thirty seven LN patients were enrolled. The correlation between the UPCR in the morning spot urine samples and urinary protein excretion in the 24-hour collections was examined using the Pearson correlation test. The best cutoffs for UPCR predicting a 24-hour protein excretion were determined with the receiver operating characteristic curve (ROC).

Results: It was found a good positive correlation between the UPCR and 24-hour protein excretion, with a correlation coefficient ( $r$ ) of 0.891 (Fig 1). The best cutoff which gave the maximum area under the curve was 0.44 for $0.5 \mathrm{~g}, 0.92$ for $1.0 \mathrm{~g}$, 2.21 for $2.0 \mathrm{~g}, 2.70$ for $3.0 \mathrm{~g}, 3.49$ for $4.0 \mathrm{~g}$ and 4.59 for $5.0 \mathrm{~g}$.

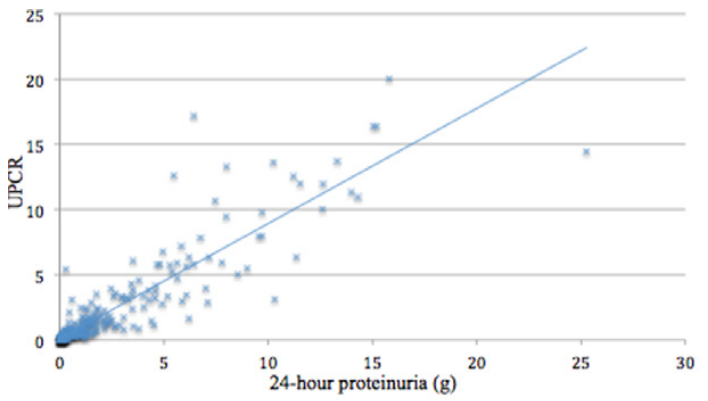

Fig. 1 Scatterplot of correlation between protein content in a 24-hour urine collection sample and morning sample of UPCR

Conclusions: The UPCR can be used as a screening test as a good predictor for proteinuria of LN patients. Also, UPCR is a valuable tool with which to monitor disease progression.

References:

[1] Bertsias GK, Tektonidou M, Amoura Z, et al. Joint European League Against Rheumatism and European Renal Association-European Dialysis and Transplant Association (EULAR/ERA-EDTA) recommendations for the management of adult and paediatric lupus nephritis. Ann Rheum Dis 2012; 71: 1771-82.

[2] Renal Disease Subcommittee of the American College of Rheumatology Ad Hoc Committee on Systemic Lupus Erythematosus Response Criteria. The American College of Rheumatology response criteria for proliferative and membranous renal disease in systemic lupus erythematosus clinical trials. Arthritis Rheum 2006; 54: 421-32.

[3] Medina-Rosas J, Yap KS, Anderson M, et al. Utility of Urinary ProteinCreatinine Ratio and Protein Content in a 24 -Hour Urine Collection in Systemic Lupus Erythematosus: A Systematic Review and Meta-Analysis. Arthritis Care Res (Hoboken) 2016; 68: 1310-9.

Disclosure of Interest: None declared

DOI: 10.1136/annrheumdis-2017-eular.5324

\section{FRI0282 CARDIOVASCULAR DAMAGE IN DECEASED PATIENTS WITH SYSTEMIC LUPUS ERYTHEMATOSUS}

I. Padjen ${ }^{1,2}$, M. Erceg ${ }^{3}$, M. Cerovec ${ }^{1,2}$, M. Mayer ${ }^{1,2}$, R. Stevanović ${ }^{3}$, B. Anić ${ }^{1,2}$ ${ }^{1}$ Department of Internal Medicine, Division of Clinical Immunology and Rheumatology, University Hospital Centre Zagreb; ${ }^{2}$ University of Zagreb, School of Medicine, ${ }^{3}$ Croatian Institute of Public Health, Zagreb, Croatia

Background: Cardiovascular comorbidities are a major contributor of damage in patients with SLE. They are driven by classical, as well as SLE-related risk factors, i.e disease activity and immunosuppressive treatment.

Objectives: We aimed to analyze cardiovascular damage (CVD) in a group of 90 deceased SLE patients regularly followed-up in a routine academic setting at our Department, and to identify features associated with accrual of CVD.

Methods: We retrospectively observed 90 SLE patients (68 females) deceased within the 2002-2011 period. All patients were $\geq 18$ years of age and Croatian residents at the time of death, fulfilling $>4$ classification criteria of the American College of Rheumatology (ACR). We identified patients with CVD, including the following components of the Systemic Lupus International Collaborating Clinics (SLICC)/ACR damage index: cardiovascular damage as defined by the index (cardiac damage), peripheral vascular damage, cerebrovascular accident, pulmonary infarction, bowel infarction and avascular necrosis. An extensive set of variables was compared between patients with and without CVD: demographics, ACR criteria at diagnosis and death, damage (according to the SLICC/ACR index) and its components one year following diagnosis and at the time of death, disease activity at diagnosis (according to the European Consensus Lupus Activity Measurements index, ECLAM), as well as features of the metabolic syndrome, smoking and immunosuppressive treatment. Frequencies were compared using the $\chi^{2}$ and Fisher's exact test, and continuous variables using the t-test and Mann-Whitney $U$ test. Variables associated with CVD in the univariate analysis were included in a multivariate logistic regression model.

Results: We identified 63/90 patients with CVD, including 46/63 (73\%) with cardiac damage, 19/63 (30\%) with peripheral vascular damage, 21/63 (33\%) 